Trauma Berufskrankh 2016 · [Suppl 2]:

18:S111-S116

DOI 10.1007/s10039-015-0056-y

Online publiziert: 26. Oktober 2015

๑) Springer-Verlag Berlin Heidelberg 2015

CrossMark

Guido Fitze

Klinik für Kinderchirurgie, Universitätsklinikum Dresden, Dresden, Deutschland

\title{
Management der Organverletzungen bei Adoleszenten
}

figsten verletzte Organ, gefolgt von Milz, Leber und Magen-Darm-Trakt. Am seltensten sind Pankreasverletzungen beobachtet worden. Diese Angaben basieren auf der Datenauswertung von Patienten mit einem stumpfen Bauchtrauma über einen Zeitraum von 20 Jahren. Dabei stellte sich heraus, dass knapp die Hälfte dieser Patienten ihr stumpfes Bauchtrauma im Rahmen eines Verkehrsunfalls erlitten hat. Im Gegensatz dazu wird in den Studien, die offenbar die Verletzung der Nieren nicht entsprechend einschließen, immer wieder angegeben, dass die Milz das am häufigsten verletzte Organ darstellt. Insgesamt ist davon auszugehen, dass bei ca. 20-25\% der stumpfen Bauchtraumata im Kindesalter eine Organverletzung im Abdominalraum vorliegt, während dies nur bei ca. $5 \%$ der Erwachsenen beobachtet wird [3].

\section{Bildgebende Diagnostik}

Grundlage der Diagnostik sind zum einen die Anamnese, der klinische Untersuchungsbefund und die Erhebung spezifischer pathologischer Laborparameter wie Erhöhung der Transaminasen bei Leberverletzungen, Erhöhung der Lipase und Amylase bei Pankreasverletzungen, eine Hämaturie bei Nierenverletzungen und eine mögliche Leukozytose bei Milzverletzungen. Zum anderen kommt der bildgebenden Diagnostik im Rahmen der Abklärung eines stumpfen Bauchtraumas eine essenzielle Bedeutung zu.

In Europa wird dabei als Primärdiagnostik die Sonographie des Abdomen klar präferiert. Hinsichtlich des Nachweises von freier Flüssigkeit im Abdominalraum ist durch diese Untersuchung eine Sensitivität von über $90 \%$ bei einer Spezifität von bis zu $97 \%$ gegeben. Dabei korreliert die Menge der nachgewiesenen freien Flüssigkeit mit der Schwere der Organverletzung, wobei große Mengen freier Flüssigkeit eher auf eine Milz- als auf eine Leberverletzung hinweisen. Umgekehrt schließt das Fehlen freier Flüssigkeit eine Verletzung parenchymatöser Bauchorgane nicht aus. Der wesentliche Vorteil der Sonographie ist die kurzfristige Wiederholbarkeit und somit das Monitoring der Befunde am Krankenbett.

Im Gegensatz zu dieser Strategie wird insbesondere in den USA als Primärdiagnostik bei Vorliegen eines stumpfen Bauchtraumas die Kontrastmittelcomputertomographie angewendet. Bei einer Sensitivität und Spezifität hinsichtlich des Nachweises einer Organverletzung von knapp $100 \%$ ist sie als die sicherste Methode und als Goldstandard anzusehen. Auf der CT-Diagnostik basiert außerdem die Schweregradeinteilung der parenchymatösen Organverletzungen des Bauchraums. Vorteile dieses Verfahrens sind ein äußerst geringer Zeitaufwand und eine kaum vorhandene Abhängigkeit der Befunde vom jeweiligen Untersucher. Somit werden Verletzungen in weniger als 2,5\% der Fälle übersehen. Im Rahmen der aktuellen Diskussion bezüglich der Strahlenexposition im Kindesalter durch eine CT-Untersuchung ist dabei unbedingt eine kindgerechte Adaptation der Untersuchungsmodalitäten zu fordern. In einer gerade publizierten niederländischen Studie [8] konnte gezeigt werden, dass bei 64 hämodynamisch stabilen Kindern nach Abdominaltrauma die CT-Untersuchung nur bei 3 Patienten zu einer Änderungen der Diagnosen und des therapeutischen Managements führte. Bei einem Patienten erfolgte nach der CT-Diagnostik eine La- 

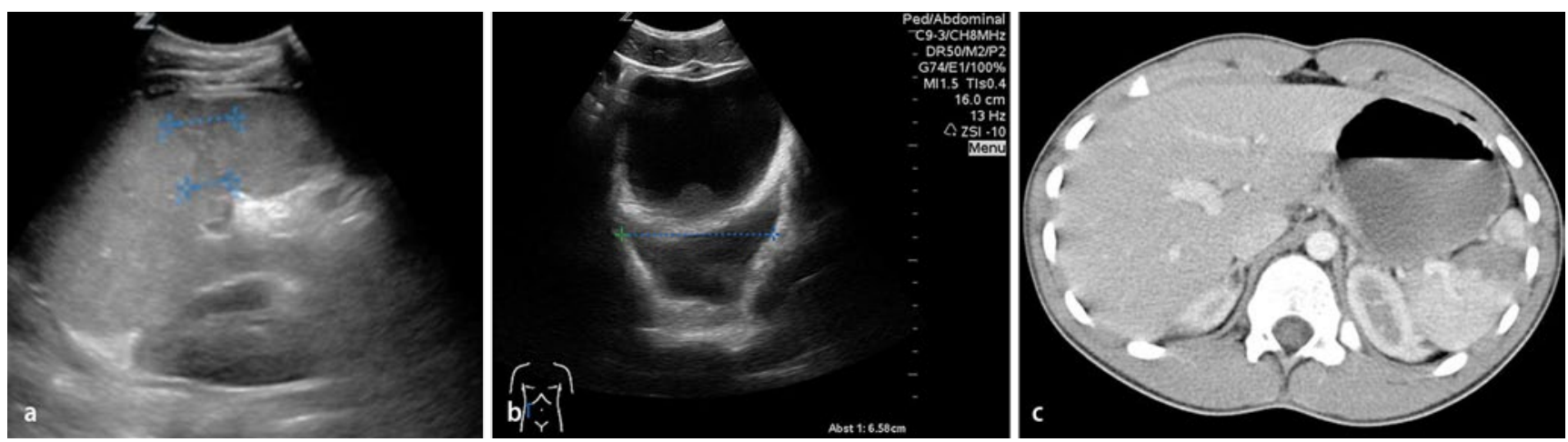

Abb. 1 \ 13-jähriger Junge, Zustand nach Sturz vom Baum aus ca. 3 m Höhe. a Sonographischer Nachweis einer zentralen Milzruptur mit wenig perisplenischer freier Flüssigkeit. b Typisch für eine Milzruptur ist die sonographisch nachgewiesene reichliche freie Flüssigkeit im Abdomen, hier retrovesikal im Rahmen der Erstuntersuchung. c Das Computertomogramm zeigt neben der Milzruptur Grad 4 eine Nierenkontusion links und eine Fraktur der 9. Rippe links
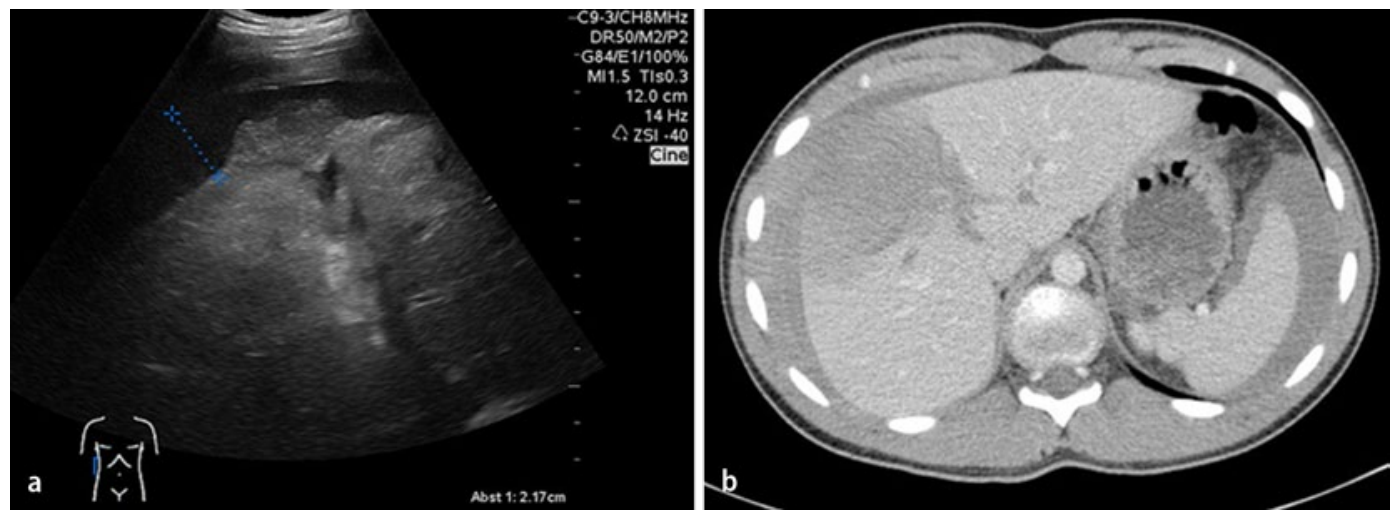

Abb. 2 × 15-jähriger Knabe ist mit dem BMX-Rad aus dem Bus gefahren, gestürzt und hat sich den Lenker in den rechten Oberbauch gerammt. a Sonographisch zeigt sich eine Leberruptur am Übergang von rechten zum linken Leberlappen mit einer ausgedehnten Kontusionszone und reichlich freier Flüssigkeit im rechten Oberbauch. b Das Kontrastmittel-CT des Abdomens stellt neben der reichlichen freien Flüssigkeit eine ausgedehnte Lazeration der Leber Grad 4 dar. Primär konservative Therapie, im Verlauf jedoch Laparotomie wegen einer Gallefistel

parotomie, da der Verdacht einer Ruptur des Duodenums bestand, die sich jedoch intraoperativ als Leberverletzung darstellte. In zwei weiteren Fällen wurde bei Nachweis einer aktiven Milzblutung eine interventionelle radiologische Embolisation durchgeführt. Die Autoren schlussfolgern, dass die Indikation zur Durchführung einer Kontrastmittel-CT im Kindesalter aufgrund einer klar formulierten, auf dem klinischen Untersuchungsbefund basierenden Fragestellung gestellt werden sollte, die eine therapeutische Konsequenz impliziert. Unter diesem Aspekt wird dem Grundsatz der rechtfertigenden Indikation zur Anwendung von Röntgenstrahlung auch auf diesem Gebiet Rechnung getragen und ist entsprechend den Prinzipien zur Durchführung der Polytraumaspirale im Kindesalter zu sehen.
Neben der diagnostischen Radiologie hat sich in den letzten Jahren auch die interventionelle Radiologie als therapeutische Option beim stumpfen Bauchtrauma im Kindesalter etabliert. In ersten Studien hat sie sich als sichere und effektive Methode bei der Behandlung von persistierenden Blutungen der Milz, der Leber und der Niere erwiesen [5]. Limitierte eigene Erfahrungen (2 Patienten) konnten diese Berichte bei der Versorgung von Nierenrupturen bestätigen.

\section{Milz- und Leberverletzungen}

Im Kindesalter gibt es für die Verletzung der parenchymatösen Oberbauchorgane Milz und Leber bei einem stumpfen Bauchtrauma verschiedene prädisponierende Faktoren. Einerseits sind beide Or- gane durch ihre anatomischen Gegebenheiten in den Zwerchfellkuppeln sehr gut fixiert. Durch den relativ hohen Flüssigkeitsanteil und den höheren Anteil an elastischen Fasern im Grundgerüst der Gewebe besitzen sie eine hohe Organspannung. Andererseits ist der Thorax im Kindesalter noch in hohem Maße verformbar. Somit kann bei einem stumpfen Bauchtrauma die kinetische Energie direkt auf beide Organe übertragen werden, was leichter zu Verletzungen führt. Bei ausgedehnten Leberverletzungen ist durch die Mitbeteiligung der großen venösen Gefäße im Kindesalter eine Mortalität von teilweise über $50 \%$ beschrieben. Das für die Milz beschriebene Phänomen der zweizeitigen Milzruptur wird zwar auch im Kindesalter kasuistisch be- 
schrieben, aber insgesamt äußerst selten beobachtet.

Bereits vor 75 Jahren hatte Wansborough am Hospital for Sick Children in Toronto die Idee, dass bei Milzverletzungen im Kindesalter nicht zwangsläufig eine Splenektomie durchgeführt werden muss. So wurde das Prinzip des nichtoperativen Managements von parenchymatösen Organverletzungen im Kindesalter entwickelt. Diese Behandlung basiert auf der Möglichkeit des Sistierens von Blutungen durch Selbstkompression im begrenzten abdominalen Raum. Voraussetzung dafür ist ein suffizientes Monitoring der Vitalparameter sowie die Möglichkeit zur engmaschigen Kontrolle von Laborparametern und dem bereits erwähnten Monitoring sonographischer Befunde am Krankenbett. Kreislaufstabilisierende Maßnahmen durch die Anwendung von Katecholaminen sowie Substitution von Flüssigkeitsverlusten bis hin zur Transfusion sind dabei fester Bestandteil der Behandlung. Bei der Transfusion von Erythrozytenkonzentraten wird eine obere Grenze von $25 \mathrm{ml} / \mathrm{kgKG}$ in den ersten $2 \mathrm{~h}$ bzw. von $40 \mathrm{ml} / \mathrm{kgKG}$ in $24 \mathrm{~h}$ toleriert. Die immer wieder postulierte Vermutung, dass im Gegensatz dazu durch ein operatives Vorgehen aufTransfusionen verzichtet werden könnte bzw. die transfundierten Mengen deutlich niedriger liegen als beim nichtoperativen Vorgehen, konnte durch Studien nicht bestätigt werden. So zeigte die Analyse von 205 Kindern mit einem Durchschnittsalter von 7 Jahren, von denen $17 \%$ operativ an einer abdominalen Organverletzung behandelt wurden, bei diesen einen signifikant höheren Transfusionsbedarf, eine längere Hospitalisierung und eine höhere Komplikationsrate [9].

In diesem Gesamtkontext hat sich in den letzten Jahrzehnten das Konzept des nichtoperativen Managements der Milzund Leberverletzungen im Kindesalter zunehmend durchgesetzt (• Abb. 1, 2 und 3). Die Effektivität und Überlegenheit dieses Konzepts konnte in vielen Studien signifikant belegt werden. Exemplarisch sei die Studie von Frumiento u. Vane aus dem Jahr 2000 [4] genannt. Von 267 Patienten mit einer isolierten Milzverletzung wurden im Zeitraum 1985-1989 nur $21 \%$ nichtoperativ versorgt. Dieser An-

Trauma Berufskrankh 2016 · [Suppl 2]: 18:S111-S116 DOI 10.1007/s10039-015-0056-y (c) Springer-Verlag Berlin Heidelberg 2015

\section{G. Fitze}

\section{Management der Organverletzungen bei Adoleszenten}

\section{Zusammenfassung}

Hintergrund. Unfälle stellen zwischen dem 1. und 20. Lebensjahr die häufigste Todesursache dar, wobei in bis zu $5 \%$ der Fälle das Abdomen beteiligt ist. Beim sog. stumpfen Bauchtrauma wird in bis zu $20 \%$ eine Organverletzung diagnostiziert; am häufigsten sind Niere und Milz betroffen, gefolgt von Leber, Pankreas und Gastrointestinaltrakt.

Diagnostik. Im Rahmen der bildgebenden Diagnostik hat im Kindesalter die Sonographie die größte Bedeutung. Die Indikation zur Kontrastmittelcomputertomographie des Abdomen muss im Gesamtkontext der Verletzung und unter dem Aspekt möglicher therapeutischer Konsequenzen kritisch gestellt werden.

Therapie. Mit wenigen Ausnahmen hat sich für diese Verletzungen im Kindesalter ein nichtoperatives Management durchgesetzt. Dieses Vorgehen ist für die isolierten Ver- letzungen der Milz und Leber evaluiert und setzt sich zunehmend auch für Verletzungen der Niere durch, wobei gelegentlich eine sekundäre Nephrektomie notwendig werden kann. Für Verletzungen des Pankreas ist bei nichtverletztem Pankreasgang eine konservative Therapie ebenfalls erfolgreich. Dagegen stellen Verletzungen des Gastrointestinaltrakts fast immer eine Indikation zur Laparotomie dar. Allgemein sollte jedoch die Laparotomie vermieden werden. Der Organerhalt hat absolute Priorität. Indikationen für eine primäre Operation sind Kreislaufinstabilität, nachgewiesene Perforation eines Hohlorgans oder Peritonitis.

\section{Schlüsselwörter}

Bauchverletzung · Gastrointestinaltrakt · Kinder · Sonographie $\cdot$ Konservative Therapie

\section{Management of organ injuries in adolescents}

\section{Abstract}

Background. Accidents are the main cause of death between the ages of 1 and 20 years. Blunt abdominal trauma occurs in approximately $5 \%$ of cases resulting in organ injury within the abdomen in about $25 \%$. The organs most involved are the kidneys and spleen followed by the liver, pancreas and the gastrointestinal (GI) tract.

Diagnostics. In the field of radiological diagnostics ultrasound is the most important procedure in children. The indications for an abdominal computed tomography (CT) scan should be critically evaluated in the context of the clinical findings and the therapeutic consequences.

Therapy. With only a few exceptions the non-operative management of abdominal organ injuries has become the established approach for children. This approach has been evaluated for spleen and liver injuries and is increasingly being applied for kidney injuries but in some rare cases a secondary nephrectomy is still necessary. For pancreatic injuries the non-operative management is also successful if the pancreatic duct is not involved. In contrast, in nearly all cases of injuries of the Gl tract a primary laparotomy is necessary but generally, a laparotomy should be avoided. The preservation of the organ is the primary target of therapy. The indications for a surgical approach are circulation instability, perforation of the GI tract and peritonitis.

\section{Keywords}

Abdominal injuries - Gastrointestinal tract . Children · Sonography · Non-operative management teil stieg in den Jahren zwischen 1990 und 1995 auf $64 \%$ an, wobei ein überproportionaler Teil in pädiatrisch spezialisierten Traumazentren konservativ behandelt werden konnte. Die Langzeitanalyse der Daten von Kindern mit isolierter Milzverletzung aus der Klinik, die das Prinzip des nichtoperativen Managements inauguriert hatte, zeigte im Zeitraum 1956-2006 einen Anstieg der nichtoperierten Kinder von $42 \%$ auf $97 \%$ [2]. Bei $99 \%$ der Patienten konnte die Milz erhalten werden. Die Transfusionen sanken von $60 \%$ auf $1 \%$ und die Mortalität bei isolierter Milzverletzung konnte von $8 \%$ auf $0,7 \%$ reduziert werden.

Analog wurde das nichtoperative Management von Milz- auf Leberverletzungen übertragen. Nellensteijn et al. [7] zeigten in ihrer Analyse aus dem Jahr 2009, 

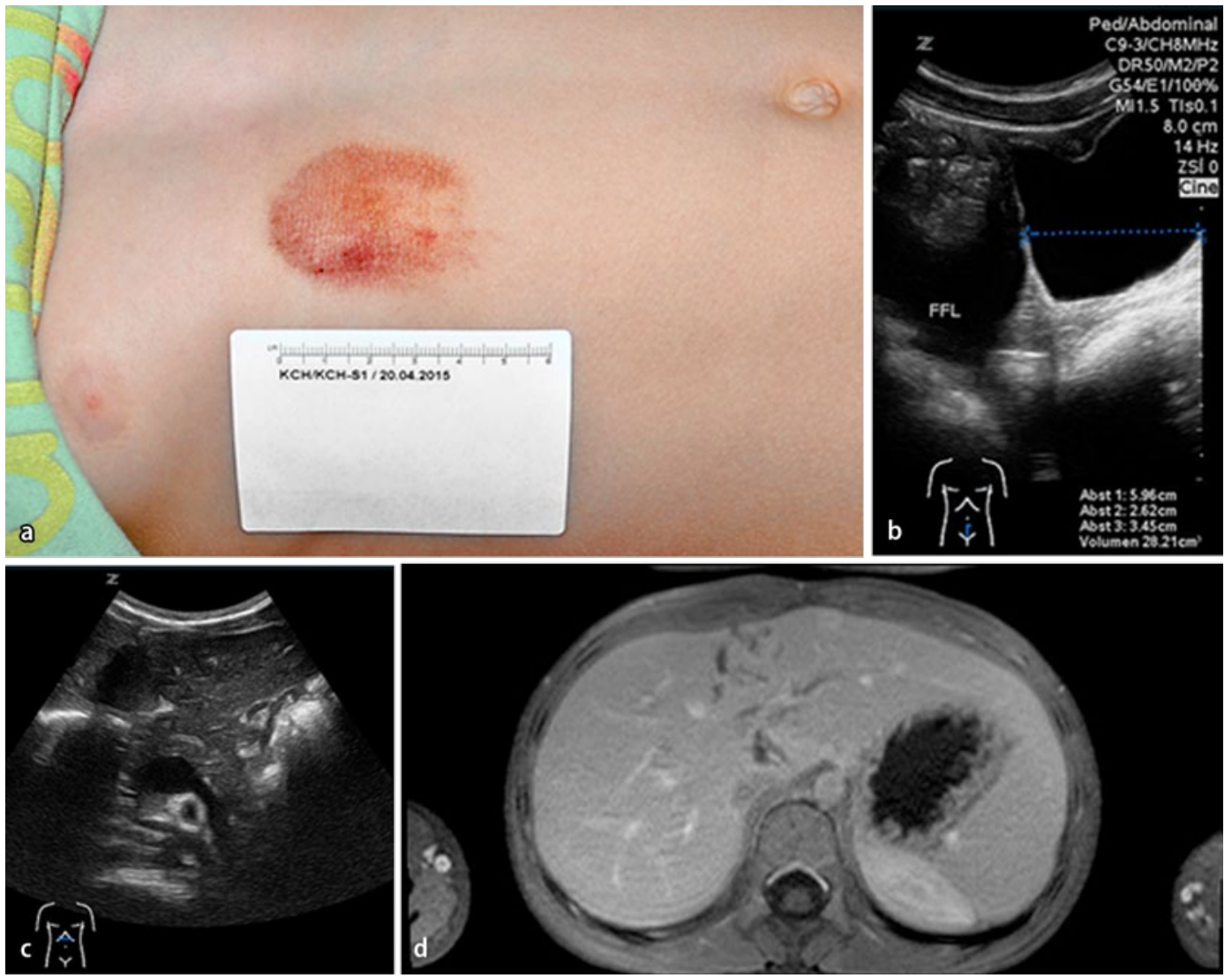

Abb. $3<8$-jähriges Mädchen ist von der Fahrradpedale abgerutscht und dabei mit dem Oberbauch auf den Fahrradlenker geprallt. a Klinisches Bild der Lenkerprellmarke im rechten Oberbauch. Die Sonographie am Unfalltag zeigt b reichlich freie Flüssigkeit retrovesikal sowie c ausgeprägte Leberläsion und Verdacht auf Pankreaskopfkontusion. d Im Magnetresonanztomogramm (T2Wichtung mit Kontrastmittel und Fettsättigung) ist die Leberruptur Grad 4 zu erkennen

dass vor $200063 \%$ aller Patienten unter 18 Jahren mit einer Leberverletzung konservativ behandelt wurden, während nach 2000 in dieser Population in $84 \%$ der Fälle primär keine Operation stattfand. Von diesen Patienten konnten $96 \%$ ohne Operation erfolgreich zu Ende behandelt werden. In einer Studie von Stylianos aus dem Jahre 2000 [10] wurde der Behandlungsverlauf von 312 Kindern mit isolierter Leber- oder Milzverletzung Grad 1-4 im Zeitraum von 2 Jahren analysiert. Dabei wurden innerhalb der ersten $48 \mathrm{~h}$ nach dem Unfall nur 4 Kinder (1,3\%) operativ versorgt. Stylianos propagiert einen sehr kurzen stationären Beobachtungszeitraum, der bei Grad-4-Verletzungen nur 5 Tage beträgt. Mit diesem Behandlungsregime kam es nur bei 6 Kindern $(1,0 \%)$ zu einer Wiederaufnahme ins Krankenhaus, wobei auch in diesen Fällen keine Operation notwendig wurde.

Sollte jedoch die Indikation für eine operative Versorgung gestellt werden, kommt dem Organerhalt höchste Priorität zu. Auch im Kindesalter ist zur Kontrolle von Blutungen das „abdominal packing“ der Resektion vorzuziehen. Viel- fältige operative Techniken finden unter dem Aspekt des Organerhalts heute Anwendung. Genannt seien die Organkompression durch die Verwendung von vorgefertigten Netzen, einfache Kapselnähte, aber auch die Applikation von Hämostyptika oder die Oberflächenkoagulation mittels Infrarot- oder Laseranwendung. Durch selektive Gefäßligaturen lässt sich eine Kontrolle der Blutung mit anschließender Organteilresektion erzielen. Die Splenektomie ist prinzipiell als Ultima Ratio anzusehen. Kleinere operative Eingriffe sowie laparoskopische Interventionen sind bei Versorgung von Milz- oder Leberverletzungen im Kindesalter entbehrlich und somit nicht indiziert.

\section{Nierenverletzungen}

Wie bereits einleitend beschrieben, ist die Niere das am häufigsten verletzte Organ bei stumpfen Bauchtraumen im Kinderalter. Insbesondere durch den anatomisch begrenzten Raum des Retroperitoneums ist für Verletzungen der Milz das Potenzial zur Selbsttamponade im Kindesalter sehr groß. Speziell unter diesem Aspekt wurde das nichtoperative Management auch auf die Verletzungen von Nieren im Kindesalter ausgeweitet. Der Erfolg dieser Behandlung wird durch vielfältige Studien belegt, zeigt aber noch eine verhältnismäßig große Variabilität hinsichtlich der Frequenz einer primären operativen Versorgung, die mit 2-33\% angegeben wird. Dabei liegt die Rate der primären Nephrektomie ebenfalls bei $2-28 \%$. Trotzdem muss darauf verwiesen werden, dass auch bei der operativen Versorgung einer Nierenverletzung im Kindesalter alle Operationstechniken zur Anwendung kommen sollten, die einen Teilorganerhalt ermöglichen. Unabhängig davon sind langfristige Verlaufskontrollen notwendig, da persistierende Perfusionsstörungen im Einzelfall auch eine Spätnephrektomie notwendig machen.

\section{Pankreasverletzungen}

Eine Verletzung des Pankreas wird im Kindesalter selten beschrieben. Jedoch ist dieses Organ durch die fixierte retroperitoneale Lage direkt vor der Wirbelsäule bei punktuell auftretender stump- 
fer Gewalt im Oberbauch für eine Verletzung im Kindesalter ebenso exponiert. Typischer Unfallmechanismus ist dabei die Lenkerverletzung durch Roller und Fahrrad. Somit ist bei klinisch sichtbarer Verletzungsmarke an der Bauchdecke prinzipiell an eine derartige Organverletzung zu denken (• Abb. 3). Klinisch imponiert diese oftmals mit zeitlicher Verzögerung, dann jedoch durch eine klassische Trias von Fieber, Leukozytose und erhöhter Amylase bzw. Lipase. Diese Laborwerte sind bei stationärer Aufnahme in bis zu $83 \%$ der Fälle bereits pathologisch erhöht. Neben der Sonographie hat hier die Kontrastmittel-CT zur diagnostischen Abklärung ihren besonderen Stellenwert.

Besonders unter dem Aspekt der Therapieentscheidung ist auch im Kindesalter, wenn möglich, eine endoskopisch retrograde Cholangiopankreatikographie (ERCP) sinnvoll. Lässt sich diagnostisch nachweisen, dass der Pankreasgang nicht verletzt wurde, ist ebenso bei Verletzungen der Bauchspeicheldrüse ein konservativer Therapieansatz Erfolg versprechend. Wird eine Läsion des Ductus pancreaticus nachgewiesen, steigt die Komplikationsrate bei konservativer Therapie auf nahezu $100 \%$ an [12]. In einer Studie von Cigdem et al. [1] wurden von 31 Pankreasverletzungen 25 konservativ behandelt. In dieser Gruppe traten bei 11 Pateinten Komplikationen auf, die in 6 Fällen eine operative Intervention notwendig machten. Somit lässt sich insgesamt einschätzen, dass der konservative Therapieansatz bei Pankreasverletzungen ohne Mitverletzung des Ductus pancreaticus Erfolg versprechend sein kann, jedoch mit einer hohen Komplikationsrate und somit möglichen sekundären operativen Versorgungen gerechnet werden muss.

\section{Verletzungen des Gastrointestinaltrakts}

Den Verletzungen des Gastrointestinaltrakts im Rahmen eines stumpfen Bauchtraumas im Kindesalter kommt insofern eine besondere Bedeutung zu, als sie primär häufig übersehen werden können. Insgesamt liegt die Frequenz derartiger Verletzungen lediglich bei 1,4-4,9\% aller Verletzungen. Es besteht eine klare Assoziation zu der Tatsache, ob Kin- der als Pkw-Insassen korrekt oder nicht korrekt angeschnallt gewesen sind. Das Risiko für das Vorliegen einer Verletzung im Bereich des Gastrointestinaltraktes ist bei nichtkorrektem Anschnallen 4-mal höher als bei korrekt angeschnallten Kindern. In beiden Populationen wird aber in über $80 \%$ der Kinder eine Gurtmarke als typisches klinisches Zeichen beschrieben [6]. Das klinische Beschwerdebild wird durch die sekundäre Peritonitis bestimmt. In den meisten Fällen kommt es zur Ausprägung eines Pneumoperitoneums, sodass hier die konventionelle Röntgendiagnostik als Abdomenübersichtsaufnahme zum Nachweis der freien Luft ihren uneingeschränkten Stellenwert hat. Zur Sicherung von retroperitonealen Verletzungen im Bereich des Duodenums ist die Kontrastmittel-CT indiziert. Therapeutisch lässt sich in diesen Fällen eine operative Versorgung der Verletzungen des Gastrointestinaltraktes nicht vermeiden. Bei Verletzungen des Duodenums sind die klassische Dekompression und Drainage indiziert. Bei Verletzungen des übrigen Intestinums finden die üblichen Operationstechniken, wie Übernähung einer Perforation, Segmentresektion oder auch die passagere Anlage eines protektiven Enterostomas, ihre Anwendung. Trotz der nicht selten verzögerten Diagnostik der Verletzungen des Gastrointestinaltrakts im Kindesalter ist die Gesamtmorbidität durch einen späteren Operationszeitpunkt nicht verschlechtert.

\section{Fazit für die Praxis}

- Im Rahmen der Diagnostik ist die Sonographie zu präferieren. Die Kontrastmittel-CT sollte abhängig vom klinischen Befund und der therapeutischen Fragestellung unter Anwendung an das Kindesalter adaptierter Untersuchungsmodalitäten erfolgen.

- Das nichtoperative Management ist die primäre Behandlungsoption für isolierte Leber- oder Milzverletzungen der Schweregrade 1-4.

- Das konservative Therapiekonzept kann auch auf Nieren- und Pankreasverletzungen ohne Läsion des Ductus pancreaticus übertragen werden.

- Verletzungen des Gastrointestinaltrakts können im Kindesalter verzö- gert diagnostiziert werden, bedingen dann aber in jedem Fall eine Laparotomie.

- Generell sollte die Laparotomie vermieden werden. Der Organerhalt hat absoluten Vorrang. Indikationen für eine primäre Operation sind Kreislaufinstabilität, nachgewiesene Perforation eines Hohlorgans oder Peritonitis.

\section{Korrespondenzadresse

Prof. Dr. G. Fitze
Klinik für Kinderchirurgie
Universitätsklinikum Dresden,
Fetscherstr. 74, 01307 Dresden
guido.fitze@uniklinikum-dres-
den.de

\section{Einhaltung ethischer Richtlinien}

Interessenkonflikt. G. Fitze gibt an, dass kein Interessenkonflikt besteht.

Dieser Beitrag beinhaltet keine Studien an Menschen oder Tieren.

The supplement containing this article is not sponsored by industry.

\section{Literatur}

1. Cigdam MK, Senturk S, Onen A et al (2011) Nonoperative management of pancreatic injuries in pediatric patients. Surg Today 41(5):655-659

2. Davies DA, Pearl RH, Ein SH et al (2009) Management of blunt splenic injury in children: evolution of the nonoperative approach. J Pediatr Surg 44:1005-1008

3. Farrath S, Parreira JG, Perlingeiro JAG et al (2012) Predictors of abdominal injuries in blunt trauma. Rev Col Bras Cir 39(4):295-300

4. Frumiento C, Vane DW (2000) Changing patterns of treatment for blunt abdominal splenic injuries: an 11-year experience in a rural state. J Pediatr Surg 35:985-989

5. Kiankhooy A, Sartorelli KH, Vane DW et al (2010) Angiographic embolization is safe and effective therapy for blunt abdominal solid organ injury in children. J Trauma 68(3):526-531

6. Lutz N, Arbogast KB, Cornejo RA (2003) Suboptimal restraint affects the pattern of abdominal injuries in children involved in motor vehicle crashes. J Pediatr Surg 38:919-923

7. Nellensteijn D, Porte RJ, van Zuuren W et al (2009) Paediatric blunt liver trauma in a Dutch level 1 trauma center. Eur J Pediatr Surg 19(6):358-361

8. Nellensteijn D, Greuter M, El Moumni M et al (2015) The use of CT scan in haemodynamically stable children with blunt abdominal trauma: look before you leap. Eur J Pediatr Surg (in press) 
9. Ozturk H, Dokucu Al, Onen A et al (2004) Non-operative management of isolated solid organ injuries due to blunt abdominal trauma in children: a fifteen-year experience. Eur J Pediatr Surg 14(1):2934

10. Stylianos S, the APSA Trauma Committee (2000) Evidence-based guidelines for resource utilization in children with isolated spleen or liver injury. J Pediatr Surg 35:164-169

11. Wessel LM, Scholz S, Jester I et al (2000) Management of kidney injuries in children with blunt abdominal trauma. J Pediatr Surg 35:1326-1330

12. Wood JH, Patrick DA, Bruny JL et al (2010) Operative vs nonoperative management of blunt pancreatic trauma in children. J Pediatr Surg 45:401406 